\title{
A Fuzzy Logic Approach to Decision Support in Medicine
}

\author{
U. Dev ${ }^{\text {a }}$ A. Sultana ${ }^{\text {b }}$, S. Talukder ${ }^{\mathrm{c}}$ and N. K. Mitra ${ }^{\mathrm{d}}$ \\ ${ }^{a}$ Department of Mathematics, Jagannath University, Dhaka, ${ }^{b}$ Department of Mathematics, Jahangirnagar \\ University, Savar, Dhaka; ${ }^{c}$ National Heart Foundation Hospital \& Research Institute, Dhaka and ${ }^{d}$ Department \\ of Mathematics, Bangladesh University of Business \& Technology, Dhaka, Bangladesh.
}

\begin{abstract}
We established an application of fuzzy set logic for the development of a decision support in medicine. In this case, we apply a model on the prognosis of a patient suffering heart failure treated with beta-blockers. It shows how the basic rules are represented in a system depending on fuzzy logic based on expert experience.
\end{abstract}

Keywords: Left ventricular diastolic diametre (LVDD), Dyspnea, Asthenia, Telediastolic, Ischemia, Servical vertibra three (S3), Asthenia, Atrio Venticular Block (AVB), Pace maker (PM), Heart failure probability (HFP).

\section{Introduction}

Medicine is one field in which the applicability of fuzzy set theory was recognized quite early in the mid-1970s. Within this field, it is the uncertainty found in the process of diagnosis of disease that has most frequently been the focus of applications of fuzzy set theory. In this section, we examine some basic issues of these applications.

With the increased volume of information available to physicians from new medical technologies, the process of classifying

The work is to develop a prototype warning system for clinical problems which is based on the assumption can be analyzed in many simple rules. The decision process of the physician that can be modeled by sets of these rules. The modeling Knowledge is usefully called "Expert Systems" and they are not new. The combination of expert system that innovate element approach with "fuzzy logic". This allows a simplification modeling and a more stable system.

In fuzzy logic all variables are described by a fuzzy set. This fuzzy set is identified by its linguistic value (for example "Ejection Fraction (EF) Low") and to evaluated the membership value (MV) of the fuzzy set. For example, patient has an EF measured during the Echocardiogram equal to $50 \%$. We show with fuzzy set "how low is an EF equal to $50 \%$ ".
Fuzzy systems contain to carry out evaluations based on domain expert experience. In this case the "Membership value of $50 \%$ to the "EF low" is 0.75 .

Every clinical variable is represented by a fuzzy set (FS) and must be fuzzyfied using corresponding functions. A membership value must be calculated for each possible value of the clinical variable. Using realistic form the evolution results of fuzzy logic systems are answers.

Each time new data feed the system with likelihood indicator. We need to know in detail a simplified and realistic rule set to achieve this task.

In particular, the nature of the clinical information and the modeling work of physicians is strongly depended on the definition of fuzzy sets. The system accesses a clinical record application for data retrieval.

The possibility to include in the new classes of system in a simple schematic way is one of the main path. There is no coding activity. This is accomplished using simultaneously fuzzy logic and conventional decision engine techniques by a step forward respect to other systems. Roughly corresponding to the probability for an information to be member of fuzzy sets supply an "activation value". These allows avoiding cascades of "if" by the activation value of Algebra. That implies the system can fire hundreds of rules and sub -rules in seconds.

\footnotetext{
* Corresponding author: E-mail:
} 
In This way the complexity is shifted from the code to the rule base content allowing problems to be configured without programming effort and enabling knowledge to be treated as ordinary digital data. The complexity of modeling knowledge is high but the activity of the technical overhead is more manageable.

It has been decided to build a system to find the degree of successful treatment of heart failure patients using betablockers administration by the actual work. By the first modeling systems for beta-blockers patients what is the probability treatment is success.

The answer will be a number from 0 (very low probability) to 1 (very high probability). This Probability is the combination of many rules like the very simple are just shown and is called Activation value (AV).

\section{Lattice}

Definition 2.1 : (Khanna, 2000) A poset $(\mathrm{L}, \leq)$ is said to form a lattice if for every $a, b \in \mathrm{L}$

Sup $\{a, b\}$ and $\operatorname{Inf}\{a, b\}$ exist in L. In that case, we write $\operatorname{Sup}\{\mathrm{a}, \mathrm{b}\}=\mathrm{a} \vee \mathrm{b},(\mathrm{a}$ join $\mathrm{b})$ $\operatorname{Inf}\{a, b\}=a \wedge b$, (a meet $b)$

Definition 2.2 : (Khanna, 2000) A non empty set L together with two binary composition (operations) $\wedge$ and $\vee$ is sade to form a Lattice if $\forall \mathrm{a}, \mathrm{b}, \mathrm{c} \in L$, the following conditions hold:

$\begin{array}{ll}\text { L 1 : Idempotency } & \mathrm{a} \wedge \mathrm{a}=\mathrm{a}, \mathrm{a} \vee \mathrm{a}=\mathrm{a} \\ \text { L 2: Commutativity } \quad: & \mathrm{a} \wedge \mathrm{b}=\mathrm{b} \wedge \mathrm{a}, \mathrm{a} \vee \mathrm{b}=\mathrm{b} \vee \mathrm{a} \\ \text { L3: Associativity } \quad: & \mathrm{a} \wedge(\mathrm{b} \wedge \mathrm{c})=(\mathrm{a} \wedge \mathrm{b}) \wedge \mathrm{c}, \\ & \mathrm{a} \vee(\mathrm{b} \vee \mathrm{c})=(\mathrm{a} \vee \mathrm{b}) \vee \mathrm{c}\end{array}$

L4: Absorption : $a \wedge(a \vee b) a, a \vee(a \wedge b)=a$. Definition 2.3: The 1- cut, ${ }^{1} \mathrm{~A}$, is called the core of $\mathrm{A}$. Definition 2.4 : The support of a fuzzy set $\mathrm{A}$ is ${ }^{{ }^{+}} \mathrm{A}$.

\section{The Fuzzy System}

The architecture of the proposed fuzzy system consists of three main blocks (Fig. 1) : the fuzzification step, the fuzzy rule base, the fuzzy inference engine. Input are given in real crisp sets and the output is a fuzzy value. The output is a real

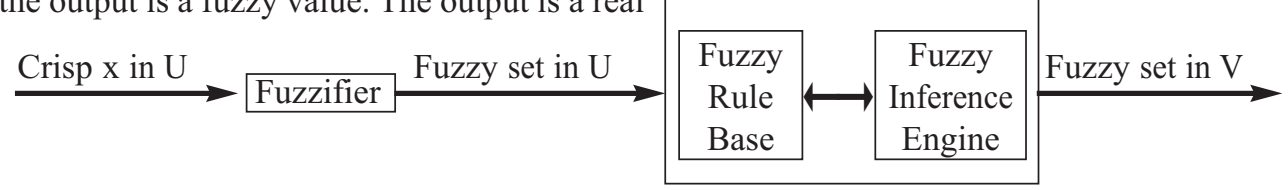

Fig. 1: Logical architecture value in the Lattice $[0,1]$ indicating the suitability degree of beta-blockers therapy.

\section{Measures of Fuzziness (Zimmermann H.J., 1996).}

Measures of fuzziness by contrast to fuzzy measures try to indicate the degree of fuzziness of a fuzzy set. A number of approaches to this end have become known. Some authors, strongly influenced by the shannon entropy as a measure of information and following de Luca and Termini (1972), consider a measure of fuzziness as a mapping $\mathrm{d}$ from the power set $\mathrm{p}(\mathrm{X})$ to $\left[0,{ }^{+}{ }_{\mathrm{O}}\right]$ satisfying a number of conditions. Others (kaufmann 1975) suggested an index of fuzziness as a normalized distance, and others (Yager 1979); Higashi and klir [1982] base their concept of a measure of fuzziness on the degree of distinction between the fuzzy set and its complement.

We shell discuss two of those measures. Suppose for both cases that the support of $\mathrm{A}$ is finite.

The first is : Let A (x) be the membership grade of the fuzzy set ${ }^{-} A$ for $\mathrm{x} \in \mathrm{X}, \mathrm{X}$ finite. It seems the measure of fuzziness $\mathrm{d}\left({ }^{-} A\right)$ should then have the following properties [de Luca and Termini 1972] :

1. $\mathrm{d}\left({ }^{-} A\right)=0$ if $^{-} A$ is a crisp set in $\mathrm{X}$.

2. $\mathrm{d}\left({ }^{-} A\right)$ assumes a unique maximum if $\mathrm{A}(\mathrm{x})=\frac{1}{2}, \forall x \in X$ 3. $\mathrm{d}\left({ }^{-} A\right) \geq \mathrm{d}\left({ }^{-1} A\right)$ if ${ }^{-1} A$ is "crisper" than ${ }^{-} A$, i.e, if ${ }^{\prime} A(x) \leq A(x)$ for $A(x) \leq \frac{1}{2}$ and ${ }^{\prime} A(x) \leq A(x)$ for $A(x) \geq \frac{1}{2}$.

4. $\mathrm{d}\left(\mathrm{C}^{-} A\right)=\mathrm{d}\left({ }^{-} A\right)$ where $\mathrm{C}^{-} A$ is the complement of ${ }^{-} A$.

The 2nd is: Knopfmacher (1975), Loo (1977), Gottwald [1979b], and others based their contributions on de Luca's and Termini's suggestion in some respects.

If ${ }^{-} A$ is a fuzzy set in $\mathrm{X}$ and $\mathrm{C}^{-} A$ its complement, then by contrast to crisp sets, it is not necessarily true that

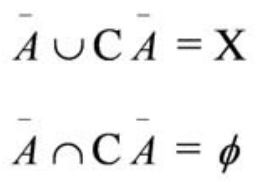


This means that fuzzy sets do not always satisfy that law of the excluded middle, which is one of their way or distinctions from traditional crisp sets. Some authors (Yager 1979; Highashi and Klir 1982) consider the relationship between $A$ and $\mathrm{C}^{-} A$ to be the essence of fuzziness.

\section{Fuzzification}

The fuzzification operator has the effect of transforming crisp data into fuzzy sets. To do this we defined five classes of Fuzzy Sets (FS) and the actual FS composing the core of this module.

\section{Classes of Fuzzy Set}

\section{Numerical Fuzzy Set}

We determined by 4 parameters $\left(\mathrm{k}_{1}, \mathrm{k}_{2}, \mathrm{k}_{3}, \mathrm{k}_{4}\right)$ with the Models numerical variables assigning membership values based on a trapezoidal function. The membership function of this fuzzy set has the following functional definition :

$$
A(x)= \begin{cases}\left(x-k_{1}\right) /\left(k_{2}-k_{1}\right) & , k_{1}<x<k_{2} \\ 1 & , k_{2} \leq x \leq k_{3} \\ \left(k_{4}-x\right) /\left(k_{4}-k_{3}\right) & , k_{3}<x<k_{4} \\ 0 & , \text { otherwise }\end{cases}
$$

The values of the four parameters depend on the particular variable.

\section{Step Fuzzy Set}

The models of discrete variables as selection from lists and check boxes assigning as activation value to each possible state of the variable.

This fuzzy set has a numerical definition of its membership function represented as a hashtable. This table depends on the variable which we are modeling.

\section{Numerical Variation Fuzzy Set}

We working on variation in time of real numerical variables taking care that the older the data are the less their weight is. Therefore the membership function of this fuzzy set has the following functional definition:

A $\left(\mathrm{x}_{1}, \ldots \ldots \ldots, \mathrm{x}_{\mathrm{n}}\right)=\left(\mathrm{x}-\mathrm{k}_{1}\right) /\left(\mathrm{k}_{2}-\mathrm{k}_{1}\right)$, if we want to detect an increasing variation of the time series.

$\mathrm{A}\left(\mathrm{x}_{1}, \ldots \ldots, \mathrm{x}_{\mathrm{n}}\right)=-\left(\mathrm{x}-\mathrm{k}_{1}\right) /\left(\mathrm{k}_{2}-\mathrm{k}_{1}\right)$, if we want to detect a lowering variation of the time series. where, $x=\sum_{i=1}^{n}\left(x_{i}-x_{i+1}\right) p_{i}, x_{i}$ is the real crisp datum at time $i, p_{i}=t^{\mathrm{i}}$ and $t \in[0,1]$ is a scaling factor which determines the weight of the i-th difference. The values of the two parameters depend on the particular variable we are modeling.

\section{Step Variation Fuzzy Set}

By observing on the variation in time of discrete variables, the fuzzy set is the result of operations on step Fuzzy Sets because the real datum is often the selection of a list or a checkbox. In this case need not to define a membership function but need to set the proper operations on fuzzy sets. In particular, considering differences, sums and weighting with respect to time. The result is the membership value for this FS. We apply the fuzzy difference Si between FS.

$\mathrm{S}_{\mathrm{i}}=\mathrm{X}_{\mathrm{i}}-\mathrm{X}_{i+1}$, if increasing variation-

$\mathrm{S}_{\mathrm{i}}=\mathrm{X}_{i+1}-\mathrm{X}_{i}$, if lowering variation

Then we define the fuzzy set Product Mi between Si and Pi

So, $\quad \mathrm{M}_{\mathrm{i}}=\mathrm{S}_{\mathrm{i}} \mathrm{P}_{\mathrm{i}}$

Where $\mathrm{P}_{\mathrm{i}}=\mathrm{t}^{\mathrm{i}}$ and $t \in[0,1]$ is a scaling factor which determines the weight of the i-th difference.

The Step Variation Fuzzy Set F is then the result of the limited sum of the fuzzy sets

$\mathrm{Mi}, \mathrm{i}=1,2, \ldots \ldots, \mathrm{n}$.

$\mathrm{F}=\mathrm{M}_{1}+\mathrm{M}_{2}+\ldots \ldots \ldots+\mathrm{M}_{\mathrm{n}}$

\section{Normality Fuzzy Set}

If numerical time series tend to a predefined normal value then the membership function has argument as the mean weighted, with respect to time, absolute difference of the real values of the time series.

$\left.\begin{array}{l}\mathrm{A}\left(\mathrm{x}_{1}, \mathrm{x}_{2}\right. \\ \text { Where }\end{array} x=\sum_{i=1}^{n}\left(x_{i}-x_{i+1}\right) p_{i}\right) / \sum_{i=1}^{n} p_{i}$

$\mathrm{x}_{\mathrm{i}}$ is the real crisp datum at time $\mathrm{i}, P_{\mathrm{i}} \in[o, 1]$ is a decreasing value which determines the weight of the $\mathrm{i}$-th deference. In which we are modeling the values of the two parameters depend on the particular variable.

\section{Fuzzy Set Example}

The input of the system represents a linguistic variable and we defined for each of them a fuzzy partition of its space through suitable fuzzy sets (see Fig. 2 \& Fig.3). The complement of each fuzzy set is always implicitly defined to com 
Table I. Fuzzy Sets definitions.

\begin{tabular}{|c|c|c|}
\hline Linguistic variable & Class & Fuzzy sets \\
\hline $\begin{array}{c}\text { SBP } \\
\text { (Systolic Blood Pressure ) }\end{array}$ & Numerical & $\begin{array}{c}\text { Low }(0.0,0.0,70.0,100.0) \\
\text { Ok }(70.0,100.0,110.0,130.0) \\
\text { high }(110.0,130.0,900.0,900.0)\end{array}$ \\
\hline SBP Progression & Numerical Variation & Lowering $(0.0,40.0,900.0,900.0)$ \\
\hline $\begin{array}{l}\text { Age big heart in family } \\
\text { history }\end{array}$ & Step & $\begin{array}{c}\text { Very young, young, adult, old } \\
(0,1,0),(1,1.0),(2,0.9),(3,0.8)\end{array}$ \\
\hline Age & Numerical & $\begin{array}{c}\text { Young }(0.0,0.0,16.0,28.0) \\
\text { Average }(16.0,28.0,42.0,50.0) \\
\text { Old }(42.0,50.0,900.0,900.0)\end{array}$ \\
\hline Heart Rate & Numerical & $\begin{array}{c}\text { Low }(0.0,0.0,40.0,55.0) \\
\text { Ok }(40.0,55.0,70.0,80.0) \\
\text { high }(70.0,80.0,900.0,900.0)\end{array}$ \\
\hline NYHA & Step & $(0,0.0),(1,0.2),(2,0.4),(3,0.7),(4,1.0)$ \\
\hline NYHA Progression & Step Variation & $(0,0.0),(1,0.2),(2,0.6),(3,0.8),(4,1.0)$ \\
\hline
\end{tabular}

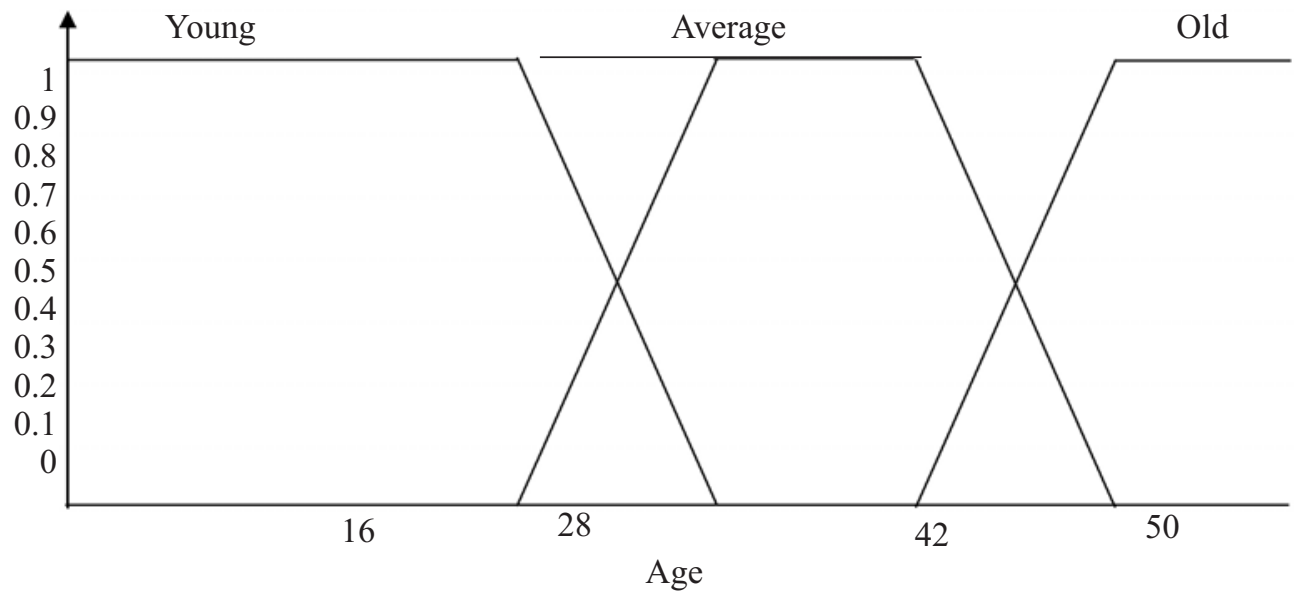

Fig. 2: Age Fuzzification

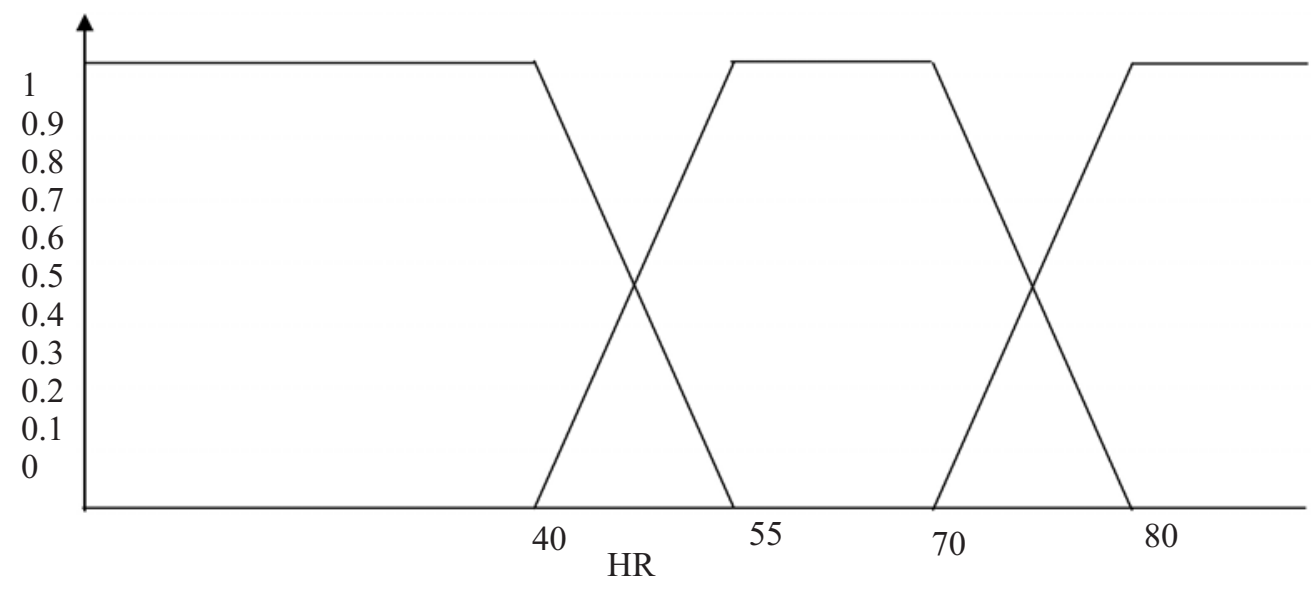

Fig. 3: Heart Rate Fuzzification 
plete the partition of the space. In following table we show the definition of a few sample fuzzy sets with the proper setting of membership function parameters.

\section{Fuzzy Rule Base}

It is easy to code them as they can be fuzzy rules modeling over Fuzzy Sets of groups. Moreover to solve an arbitrary Problem Fuzzy Rules operators can be other Fuzzy Rules.

We have defined a fuzzy rule as a list of n Fuzzy Sets and corresponding logical operators connecting them. NOT, AND and OR are the basic logical operators, connecting in a lexicographical way.

In the following table it is shown how logical operators have been modeled.

Table II: Implemntation of logical operators.

\begin{tabular}{|c|c|}
\hline Operator & Implementation \\
\hline NOT & $\bar{A}(x)=1-A(x)$, (Complement) \\
\hline AND (t-norm) & $(A \quad B)(x)=\min [A(x), B(x)],($ Minimum $)$ \\
\hline OR (t-conorm) & $(A \quad B)(x)=\max [A(x), B(x),($ Minimum $)$ \\
\hline
\end{tabular}

The output of a Fuzzy Rule is a real number in the lattice $[0,1]$ corresponding values of each Fuzzy Set corresponding to the actual medical data read for that patient.

\section{Rule Base}

A sample list of rules follows. More than 5 approximation rules are needed to model our complex problem. Hence the fuzzy approach is simple to implement. The following rule is characterised by a set of linguistic statements based on knowledge of expert.

1. IF rule 2 AND NOT rule 6 AND NOT rule 5 AND rule 4 AND NOT rule 7 AND rule 8 AND NOT rule 9 AND NOT rule 10 THEN 'BETA-BLOCKERS ok'.

2. IF rule 3 OR EF low THEN 'Patient has heart failure'.

3. IF LVDD high OR Telediastolic volume high THEN 'Big Heart'.

4. IF Ischemia in family for relative 1 AND Degree of family relationship for relative 1 THEN 'Ischemia from family history'.

5. IF NYHA class progression OR Dyspnea Progression OR Abdominal discomfort Progression OR Asthenia
Progression OR EF lowering OR SBP lowering THEN 'HEP not OK for beta-blockers'.

6. IF Big heart in family for relative 1 AND Age big heart in family for relative 1 AND Degree of family relationship for relative 1 AND Age young THEN 'Big heart from family history and low Age not OK for 'beta-blockers'.

7. IF HR low THEN 'beta-blockers NOT ok'.

8. IF NOT SBP low THEN 'beta-blockers ok'.

9. IF Jugular Turgor 45o OR S3 OR Na high OR Dyspnea OR Abdominal discomfort OR Asthenia OR NYHA THEN 'counter-indications'.

10. IF AVB AND NOT PM implanted THEN 'beta-blockers not OK'.

\section{The Inference Engine}

The inference engine is the code that fires the rules in a given order and provides the fuzzy output according to chosen inference model. The firing order is not connected by logical relations but relevant only for rules. The rules contained as operators in other rules are fired in a recursive way, Deadlock situations are avoided as a limit in the depth of recursive calling is fixed and checked at every iteration. We are applying the inference model as the Tsukamoto's one. Obtaining the fuzzy output of such a knowledge base consists from the procedure for the following three steps.

1. to find the firing level of each of the rules

2. to find the output of each of the rules

3. aggregate the individual rule outputs to obtain the overall system output.

\section{Experimental Results}

The system was developed during 2001 and 2002. From the clinical record software we take the data collection which is based upon the system. By the fuzzy logic system the data which was collected during routine treatment of patients.

All software was coded using the Mathematical Programming language and can be used on internet network environments.

The out come of the processing is presented as HTML text reporting each step of the logic process, and hyperlink to the relevant scientific references. 
The following Fig. 4 shows an expert of the output.

FUZY RULE number: 10 "patient has heart failure".

Its depth level is 2 and it derives from rule 10 Reference document: description doc. 1

\begin{tabular}{c|c|c|c|c|c}
\hline F.S. & Description & value & date & M.V. & Rule \\
\hline 120 & EF low & $40(\%)$ & $07-08-2009$ & 1,000 & 10 \\
& & & $18: 17: 19$ & & \\
\hline
\end{tabular}

Conclusions of Fuzzy rule number 10.

The rule is described by the logic statement: rule 30 or EF low and its evaluation is: 1,000

Fuzzy Rule number: 30 "Big Heart".

Its depth level is 3 and it derives from rule 10 Reference document: description doc. 2

\begin{tabular}{c|c|c|c|c|c}
\hline F.S. & Description & Value & Date & M.V. & Rule \\
\hline 125 & LVDD high & $70(\mathrm{~mm})$ & $\begin{array}{c}07-08-2009 \\
18: 17: 19\end{array}$ & 1,000 & 30 \\
\hline 135 & $\begin{array}{c}\text { Telediastolic } \\
\text { Volume high }\end{array}$ & N/A & N/A & N/A & 30 \\
\hline
\end{tabular}

Conclusions of Fuzzy Rule number 30.

The rule is described by the logic statement: LVDD high OR Telediastolic volume high and its evaluation is : 1,000.

Fig. 4: Sample output

The tests have been carried out on two patients, who have been thoroughly studied and the results are in very good agreement with the clinical evaluation of the physicians

\section{Conclusion}

For several reasons the application of systems as the one reported in this work in a real clinical activity is not foreseen in the near future. The results as in other similar works are promising and encourage us to continue development. There are fields where immediate applications are possible, as education, simulation, and optimisation application. The software feature of this system allows Application Service Provisioning very promising for commercials applications.

\section{References}

Aversa F., Gronda E., Pizzuti S. and Aragno C., A fuzzy logic approach to decision support in Medicine. Kell s.r.l., Via E.Q. Visconti, 800193 Rome Italy, Clinical Cardiology and Heart Failure Unit, Dept. of Cardiology, Humanitias clinical Institute Via A. Manzoni, 56,20089, Rozzano, Italy.

Chen S. M. (1994). A weighted fuzzy reasoning algorithm for medical diagnosis, Decision Support Systems, 11: 37-43.

Driankov D., Hellendoom H. and Reinfronk M. (1993). An Introduction to Fuzzy Control (Springer Verlag, Berlin).

Dubois D. and Prade H. (1980). Fuzzy Sets and Systems : Theory and Applications (Academic Press, London).

Dubois D. and Prade H. (1988). Possibility Theory (Plenum Press, New York).

Khanna V. K. (2000). Lattices and Bollean Algebras. 1st Ed. (S.N. Press, Delhi) 1994.
Kosko B. (1992). Fuzzy Systems as Universal approximators, in : Proc. IEEE 1992 Int. Confrence Fuzzy Systems, San Diego, 1153-1162.

Kosko B. (1992). Neural networks and fuzzy systems, prentice Hall, New Jersey.

Takagi T. and Sugeno M. (1985). Fuzzy identification of systems and its applications to modeling and control, EEE Trans. Syst. Man Cybernet, 116-132.

Yager R. R. and Filev D. (1994). Essentials of fuzzy Modeling and control (Wiley, NewYork).

Zadeh L. A. (1979). A theory of approximate reasoning, In : J. Hayes, D. Michie and L.I. Mikulich eds, Machine, Intelligence, Vol. 9 (Halstead Press, New York) 147-194.

Zadeh L. A. (1975). Concept of a linguistic variable and its application to approximate reasoning I, II, III, Information Sciences, 8 (1975). 199-249, 301-357; 9: 43-80.

Zadeh L. A. (1978). Fuzzy sets as a basis for a theory of Possibility, Fuzzy Sets and Systems, 1: 3-28.

Zadeh L.A. (1965). Fuzzy Sets, Information and Control, 8: 338-353.

Zimmermann H. J. (1987). Fuzzy sets, Decision Making and Expert Systems (Kluwer Academic Publisher, Boston).

Zimmermann H. J. (1996). Fuzzy set theory and its applications (Kluwer Academic Publishers, Dordrecht) 1991.

Received: April 05, 2009;

Accepted : August 04, 2010 